\title{
Episodic ventriculomegaly due to hypernatremia mimicking shunt malfunction: case report
}

\author{
${ }^{*}$ Sarah C. Jernigan, MD, MPH, ${ }^{1}$ Scellig S. D. Stone, MD, PhD, ${ }^{1}$ Joshua P. Aronson, MD, ${ }^{2}$ \\ Melissa Putman, MD, ${ }^{3}$ and Mark R. Proctor, MD ${ }^{1}$
}

\begin{abstract}
Departments of ${ }^{1}$ Neurosurgery and ${ }^{3}$ Medicine, Division of Endocrinology, Boston Children's Hospital; and ${ }^{2}$ Department of Neurosurgery, Massachusetts General Hospital, Boston, Massachusetts
\end{abstract}

\begin{abstract}
Patients with shunted hydrocephalus presenting with altered mental status and ventriculomegaly are generally considered to be in shunt failure requiring surgical treatment. The authors describe a case of shunted hydrocephalus secondary to a disseminated neuroectodermal tumor in a pediatric patient in whom rapid fluctuations in sodium levels due to diabetes insipidus repeatedly led to significant changes in ventricle size, with invasively confirmed normal shunt function and low intracranial pressure. This clinical picture exactly mimics shunt malfunction, requires urgent nonsurgical therapy, and underscores the importance of considering serum osmolar abnormalities in the differential diagnosis for ventriculomegaly.

http://thejns.org/doi/abs/10.3171/2015.3.PEDS1526
\end{abstract}

KEY WORDS hypernatremia; ventriculomegaly; hydrocephalus; shunt

$\mathrm{W}$ HEN a child with a ventriculoperitoneal shunt presents with symptoms such as nausea, vomiting, irritability, or altered level of consciousness, the possibility of a shunt malfunction should be investigated. The clinical evaluation almost always includes imaging of the ventricular system to look for dilation compared with baseline. ${ }^{2}$ In turn, increased ventricular size is generally interpreted as a shunt malfunction, as few conditions can mimic its presentation and imaging.

Although new ventriculomegaly in a shunt-treated patient generally indicates shunt malfunction, this is not the only cause of increased ventricular size. Subacute ventriculomegaly can result from cerebral volume loss in certain situations-for instance, following brain insults such as infection or trauma, in certain neurodegenerative conditions, and due to systemic factors such as high-dose glucocorticoid exposure and end-stage renal disease. ${ }^{6,16}$ In addition, 2 cases have been reported in which an isolated episode of hypernatremia in patients with shunted hydrocephalus caused by acute shifts in brain parenchymal water content, with corresponding transient ventriculomegaly and clinical signs of decreased level of consciousness. ${ }^{3,7}$ We expand the modest literature on transient ventriculomegaly by adding the current report of shunted hydrocephalus due to a brain tumor in a pediatric patient in whom rapid fluctua- tions in sodium levels due to diabetes insipidus repeatedly led to significant changes in ventricle size, with invasively confirmed normal shunt function and low intracranial pressure (ICP) mimicking shunt malfunction.

\section{Case Report}

Medical History

A 4-year-old boy with a recent history of diabetes insipidus and shunted hydrocephalus was transferred to our institution from Egypt. He had undergone shunt placement prior to transfer for ventriculomegaly, and contrastenhanced MRI of the brain and spine revealed widespread leptomeningeal enhancement. Upon arrival, examination of a right temporal lobe biopsy sample demonstrated a diffuse neuroectodermal neoplasm with neurocytoma features, restricted to the leptomeninges and Virchow-Robin spaces. Tumor cells focally expressed neuronal and glial markers, and no anaplastic features. The MIB-1 proliferative index was estimated at 7\%, and FISH analysis revealed 3' BRAF duplication. The leptomeningeal dissemination and involvement of the pituitary stalk were thought to be the source of the patient's diabetes insipidus. After initial shunt placement in Egypt, his parents reported that a malfunction had occurred that presented as intractable

ABBREVIATIONS DDAVP = desmopressin acetate; FOR = frontal and occipital horn ratio; ICP = intracranial pressure.

SUBMITTED January 14, 2015. ACCEPTED March 23, 2015.

INCLUDE WHEN CITING Published online July 17, 2015; DOI: 10.3171/2015.3.PEDS1526.

DISCLOSURE The authors report no conflict of interest concerning the materials or methods used in this study or the findings specified in this paper.

* Drs. Jernigan and Stone contributed equally to this work. 
vomiting and the patient underwent a shunt revision. The details of this revision, including ICP measurement and serum sodium concentration, were unobtainable.

\section{History of Presenting IIIness and Perioperative Course}

The patient had been doing well at home for several months, undergoing chemotherapy with vincristine and carboplatin, until he presented with several generalized tonic-clonic seizures. A head CT scan on admission demonstrated no ventriculomegaly, and his shunt was thought to be functioning normally. Laboratory workup revealed a serum sodium level of $146 \mathrm{mEq} / \mathrm{L}$. A brain MRI scan was planned to reassess his tumor burden and look for an etiology for the seizures. In the intervening 6 hours, the patient became increasingly lethargic, and while undergoing MRI, he became unresponsive (Glasgow Coma Scale score of 8). Imaging revealed marked ventriculomegaly, which prompted emergency operative shunt exploration. Intraoperatively, no clear obstruction was found, his ICP was too low to measure with a manometer, and there was good flow of CSF through his ventricular catheter. Laboratory samples were sent during the time of his deterioration in the MRI scanner; once the patient was in the operating room, we noted a serum sodium of $160 \mathrm{mEq} / \mathrm{L}$. Postoperative CT scanning demonstrated persistently enlarged ventricles, and a repeat testing of his serum sodium level showed $167 \mathrm{mEq} / \mathrm{L}$. He was started on a vasopressin infusion, and his sodium level was slowly corrected. Two days later, his serum sodium level was $138 \mathrm{mEq} / \mathrm{L}$, and a CT scan demonstrated decreased ventricle size.

\section{Postoperative Course}

Over the following 2 weeks, he remained in the hospital with fluctuating sodium levels as his intravenous vasopressin regimen was switched to oral dosing. Fluctuations in his serum sodium reliably correlated to his clinical examination status and, more surprisingly, to his ventricular size on imaging studies (Fig. 1). Changes in neurological examination status and imaging findings were not dependent on relative changes in the serum sodium but on absolute serum sodium values, with increased ventricular size and somnolence occurring when his sodium exceeded 145 $\mathrm{mEq} / \mathrm{L}$ (Fig. 2). We calculated the frontal and occipital horn ratio ${ }^{14}$ (FOR) for each scan and plotted these ratios with their corresponding serum sodium concentration (Fig. 3). There was a strong positive correlation between the FOR and serum sodium concentration $(\mathrm{r}=0.94, \mathrm{n}=$ $12, \mathrm{p}<0.01)$. Once the patient's sodium level was well controlled, he was discharged to home while taking an oral DDAVP (desmopressin acetate) regimen; his ventricles returned to baseline size. Follow-up MRI scans 2 and 6 months later demonstrated near-slit ventricles, with corresponding serum sodium values of 137 and $140 \mathrm{mEq} / \mathrm{L}$, respectively. At no time did any MRI scans demonstrate new or evolving ischemic changes, including of the hypothalamus and brainstem.

\section{Second Presentation}

The patient presented to the emergency department 1 week after the second follow-up MRI study with somnolence and decreased responsiveness. He had been at the beach 2 days prior, then became more sleepy with decreased oral intake, and missed his morning dose of DDAVP. Head CT scans revealed ventriculomegaly, and his serum sodium level was $153 \mathrm{mEq} / \mathrm{L}$. His shunt was accessed via a transcutaneous shunt tap, and his ICP was very low, with good flow of CSF. He was admitted to the ICU, placed on a vasopressin infusion, and slowly returned to his neurological baseline as his serum sodium normalized. A repeat CT scan 24 hours later demonstrated decreased ventricular size with a serum sodium level of $140 \mathrm{mEq} / \mathrm{L}$.

\section{Discussion}

Hypernatremia is a physiological disturbance defined as a concentration of serum sodium greater than 150 $\mathrm{mEq} / \mathrm{L} .{ }^{8}$ The sodium concentration is regulated by water homeostasis, which is controlled by thirst and antidiuretic hormone release. With an increasing extracellular sodium concentration, plasma tonicity increases, causing movement of water out of cells. This results in cellular dehydration and crenulation. The brain is particularly vulnerable to fluid shifts associated with hyperosmotic hypernatremia, which is indeed a treatment for brain swelling. ${ }^{4}$ An animal study by Finberg and colleagues demonstrated subdural and intracerebral hemorrhages in experimentally induced hypernatremia. Cellular dehydration caused shrinkage of the brain, resulting in tearing of the cerebral bridging veins and subdural hematomas. ${ }^{9}$ In these studies, the cellular dehydration and shrinkage caused a relative loss in brain parenchymal volume and increase in cerebral spinal fluid spaces, resulting in ventriculomegaly. Several studies have shown that clinical imaging in patients with severe prolonged hypernatremia revealed microhemorrhages and diffuse white matter edema along with increased ventricular size..$^{5,12,13}$

There are 3 previous case reports of transient ventriculomegaly in patients with shunted hydrocephalus in hyperosmolar states, 2 of which involve hypernatremia., ${ }^{3,10}$ Each describes 1 documented episode of ventriculomegaly that improved once the osmolar imbalance was corrected. In the single prior pediatric case involving hypernatremia, a 3-year-old child's elevated serum sodium concentration resulted from severe dehydration, and surgery was avoided based on the results of a shunt tap. ${ }^{7}$ In another case of hypernatremia, an adult with diabetes insipidus secondary to a brain tumor presented with neurological decline and ventriculomegaly and was rushed to surgery. The shunt was functioning, and hypernatremia was discovered. Of note, the patient was undergoing chemotherapy, and desmopressin was being temporarily withheld, predisposing the patient to the hypernatremia. ${ }^{3}$ In the third case the authors described a 15-year-old patient with myelomeningocele-related shunted hydrocephalus who presented with a short history of altered mental status, vomiting, and ventriculomegaly. ${ }^{10}$ At presentation the patient was in diabetic ketoacidosis and in a hyperglycemic, hyperosmolar state. Although the patient's ICP was never measured, symptoms and ventriculomegaly completely resolved within 24 hours of correcting the hyperosmolar state.

Our case is unique because the child showed a repeated pattern correlating variations in ventricular size with serum sodium levels, and twice he had invasive confirmation of 


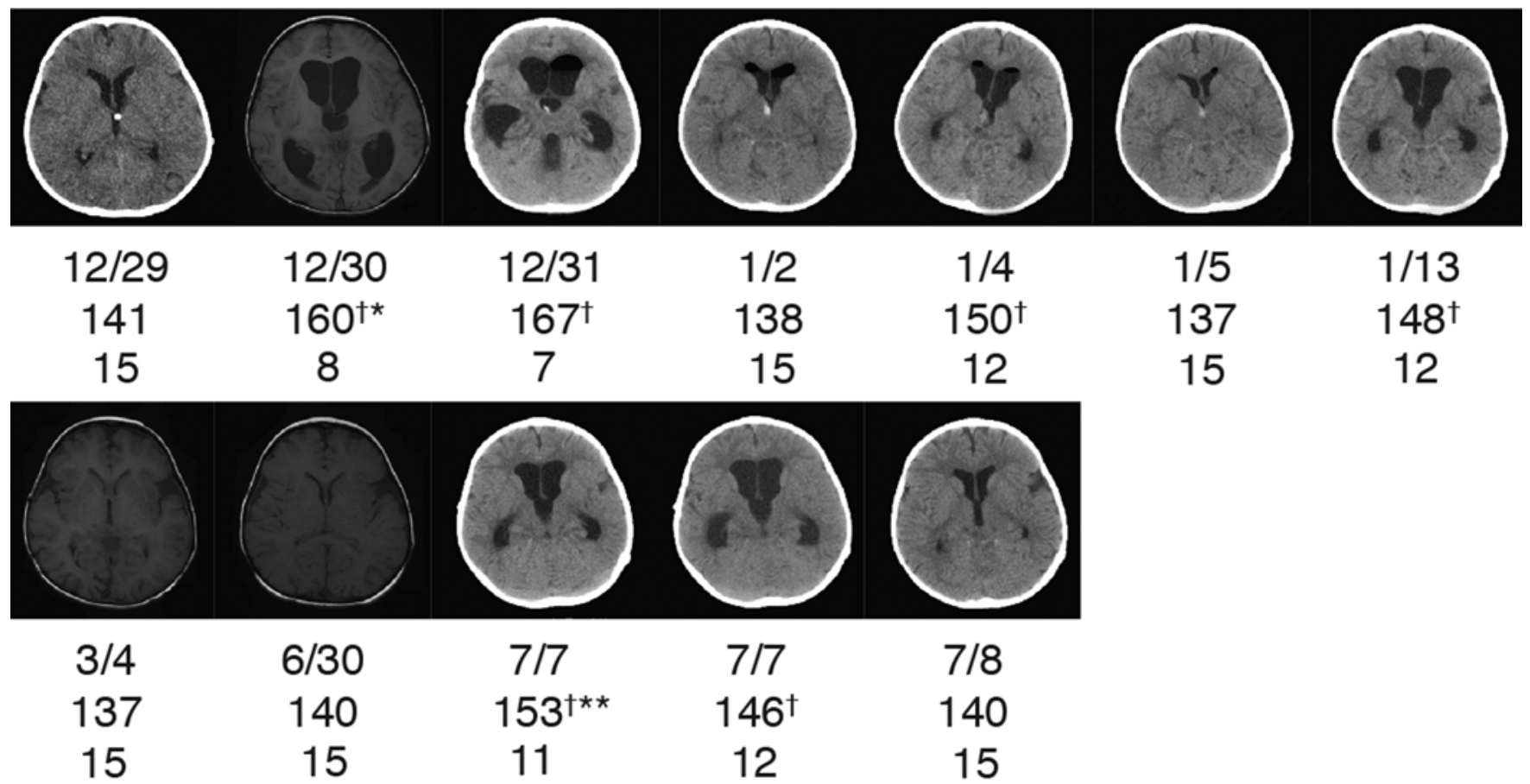

FIG. 1. Series of CT and MR brain images over the course of repeated hospitalizations. The date (upper values), serum sodium concentration (in $\mathrm{mEq} / \mathrm{L}$ [center values]), and Glasgow Coma Scale score (lower values) are listed below each image. Sodium level with an asterisk indicates surgical exploration of a shunt with low pressure, double asterisks indicates a shunt tap with low pressure, and the dagger indicates altered mental status.

shunt function and low ICP in the setting of transient ventriculomegaly associated with hypernatremia. The child's diabetes insipidus predisposed him to recurrent electrolyte imbalances, to which he was neurologically very sensitive, as indicated by his decreased level of arousal with relatively mild elevations in serum sodium of $145-150 \mathrm{mEq} / \mathrm{L}$.
Based on the experience gained from the first episode of hypernatremia-induced symptomatic ventriculomegaly, we were able to avoid unnecessary surgery during our patient's second hospital stay by confirming low ICP and proximal catheter patency via a shunt tap and hypernatremia via expedited serum testing. Indeed, in the absence of

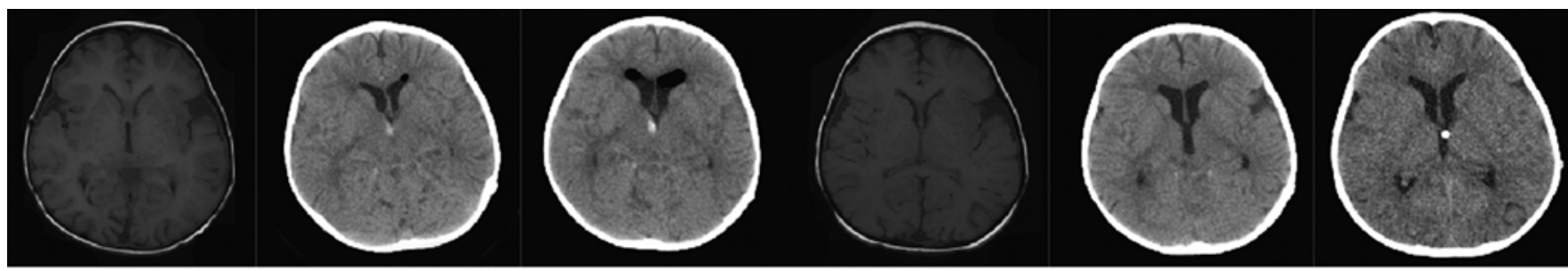

$\begin{array}{llllll}137 & 137 & 138 & 140 & 140 & 141\end{array}$

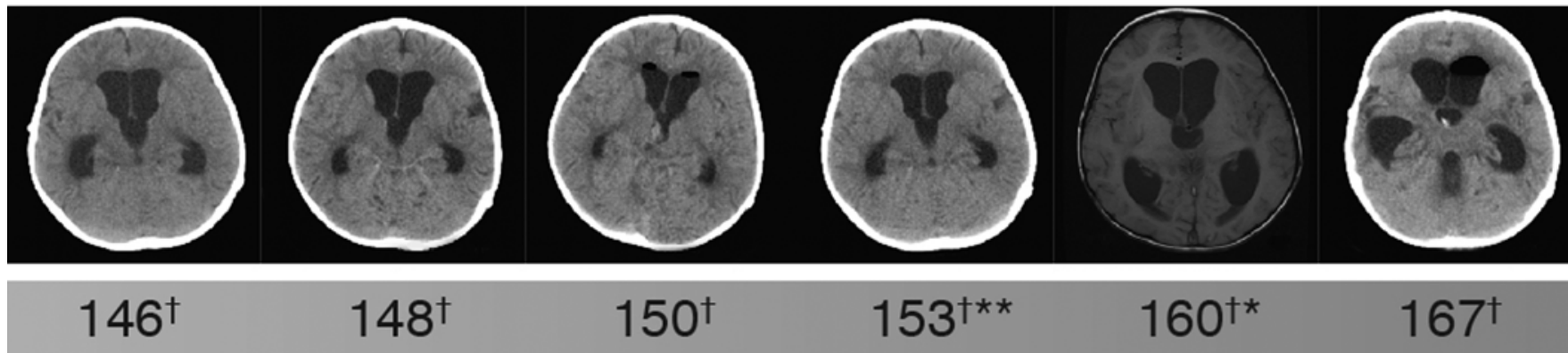

FIG. 2. Same images from Fig. 1, sorted by serum sodium concentration (in $\mathrm{mEq} / \mathrm{L}$ ). Sodium level with an asterisk indicates surgical exploration of a shunt with low pressure, double asterisks indicates a shunt tap with low pressure, and the dagger indicates altered mental status. 


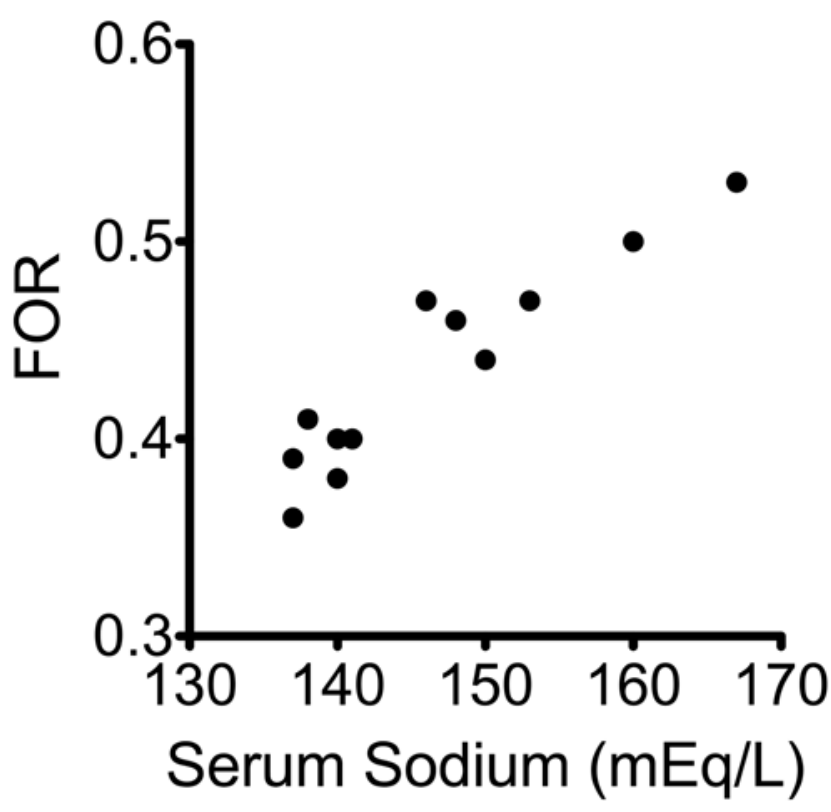

FIG. 3. The FOR plotted with serum sodium concentration derived from the episodes depicted in Figs. 1 and 2.

more information surrounding his initial diagnosis of hydrocephalus, it is interesting to speculate as to whether he actually needed a shunt. It is plausible for a patient like this to be undergo shunt surgery in response to symptomatic ventriculomegaly without true hydrocephalus.

The etiology of hydrocephalus in this case-leptomeningeal dissemination of a neuroectodermal tumor-may also play a role in the development of marked ventriculomegaly with hypernatremia. Altered brain elasticity has been proposed as a mechanism for low-pressure ventriculomegaly in some shunt-treated patients, including cases resulting from ventriculitis, tumors, and hemorrhage. ${ }^{1,11,15}$ Reduced brain cellular and extracellular water content is thought to further alter the brain's viscoelastic properties, leading to ventricular dilation without raised ICP. Symptoms may arise from distorted brain tissue and impaired perfusion. Encouraging water to reenter the brain parenchyma may restore normal brain compliance and, in turn, promote a return to normal ventricular size. Indeed, manipulations aimed at increasing brain turgor, such as elevating jugular vein pressure, can lead to a resolution of ventriculomegaly and symptoms in some cases.

Although it is important to investigate the possibility of shunt malfunction in any shunt-treated patient with an altered level of consciousness, this report underscores the importance of assessing serum electrolytes and osmolality, especially in patients at risk, such as those with brain tumors affecting the neuroendocrine axis. Our case demonstrates that symptomatic marked ventriculomegaly in a shunt-treated child can be caused by hypernatremia yet present with the same clinical picture as shunt malfunction, and can resolve with medical correction of the electrolyte imbalance. Pediatricians, neurologists, and neurosurgeons should be aware of the potentially dramatic effect that hypernatremia can have on cerebral volume and consider serum osmolar abnormalities in the differential diagnosis for ventriculomegaly.

\section{References}

1. Akins PT, Guppy KH, Axelrod YV, Chakrabarti I, Silverthorn J, Williams AR: The genesis of low pressure hydrocephalus. Neurocrit Care 15:461-468, 2011

2. Albright AL, Pollack IF, Adelson PD: Operative Techniques in Pediatric Neurosurgery. New York: Thieme, 2001

3. Andres RH, Pendharkar AV, Kuhlen D, Mariani L: Ventricular enlargement due to acute hypernatremia in a patient with a ventriculoperitoneal shunt. J Neurosurg 113:82-84, 2010

4. Conley SB: Hypernatremia. Pediatr Clin North Am 37:365-372, 1990

5. Cozzens JW, Chandler JP: Increased risk of distal ventriculoperitoneal shunt obstruction associated with slit valves or distal slits in the peritoneal catheter. J Neurosurg 87:682686, 1997

6. Damsted SK, Born AP, Paulson OB, Uldall P: Exogenous glucocorticoids and adverse cerebral effects in children. Eur J Paediatr Neurol 15:465-477, 2011

7. Fahrbach J, Rozzelle CJ: Transient ventriculomegaly in a child presenting with hypernatremia. Case report. J Neurosurg 104 (3 Suppl):192-194, 2006

8. Finberg L, Kravath RE, Hellerstein S: Water and Electrolytes in Pediatrics: Physiology, Pathology, and Treatment, ed 2. Philadelphia: Saunders, 1993

9. Finberg L, Luttrell C, Redd H: Pathogenesis of lesions in the nervous system in hypernatremic states. II. Experimental studies of gross anatomic changes and alterations of chemical composition of the tissues. Pediatrics 23:46-53, 1959

10. Gruber TJ, Rozzelle CJ: Transient ventriculomegaly in an adolescent presenting with shunted hydrocephalus, diabetic ketoacidosis, and hyperglycemia. Pediatr Neurosurg 44:496-500, 2008

11. Lesniak MS, Clatterbuck RE, Rigamonti D, Williams MA: Low pressure hydrocephalus and ventriculomegaly: hysteresis, non-linear dynamics, and the benefits of CSF diversion. Br J Neurosurg 16:555-561, 2002

12. Machino T, Yoshizawa T: Brain shrinkage due to acute hypernatremia. Neurology 67:880, 2006

13. Musapasaoglu H, Agildere AM, Teksam M, Tarcan A, Gurakan B: Hypernatraemic dehydration in a neonate: brain MRI findings. Br J Radiol 81:e57-e60, 2008

14. O'Hayon BB, Drake JM, Ossip MG, Tuli S, Clarke M: Frontal and occipital horn ratio: A linear estimate of ventricular size for multiple imaging modalities in pediatric hydrocephalus. Pediatr Neurosurg 29:245-249, 1998

15. Pang D, Altschuler E: Low-pressure hydrocephalic state and viscoelastic alterations in the brain. Neurosurgery 35:643656,1994

16. Schnaper HW, Cole BR, Hodges FJ, Robson AM: Cerebral cortical atrophy in pediatric patients with end-stage renal disease. Am J Kidney Dis 2:645-650, 1983

\section{Author Contributions \\ Conception and design: Proctor, Jernigan. Acquisition of data: Proctor, Jernigan, Stone, Aronson. Analysis and interpretation of data: all authors. Drafting the article: all authors. Critically revising the article: all authors. Reviewed submitted version of manuscript: all authors. Approved the final version of the manu- script on behalf of all authors: Proctor. Statistical analysis: Stone. Administrative/technical/material support: Proctor. Study super- vision: Proctor.}

\section{Correspondence}

Mark R. Proctor, Department of Neurosurgery, Boston Children's Hospital, Hunnewell 2, 300 Longwood Ave., Boston, MA 02115. email: mark.proctor@childrens.harvard.edu. 